\title{
Seismo Electromagnetics and Earthquake Prediction: History and New directions
}

\author{
M. Hayakawa ${ }^{1,2,3}$ \\ ${ }^{1}$ Hayakawa Institute of Seismo Electromagnetics, Co. Ltd. (Hi-SEM), UEC Alliance Center, \#521, 1-1-1 Kojima- \\ cho, Chofu Tokyo 182-0026, Japan \\ ${ }^{2}$ University of Electro-Communications (UEC), Advanced Wireless \& Communication research Center, 1-5-1 \\ Chofugaoka, Chofu Tokyo 182-8585, Japan \\ ${ }^{3}$ Geoscent Technologies Inc., 2-8-11 Akasaka, Minato-ku, Tokyo, 107-0052, Japan
}

\begin{abstract}
This review will focus on earthquake (EQ) precursor studies for EQ prediction. Among the long-, medium-, and short-term EQ predictions, the most meaningful are short-term predictions because of their immediate effect on human lives. Investigations of EQ precursors were very scarce before the 1995 Kobe EQ, but various kinds of precursors were reported for this disastrous Kobe EQ. Then intensive observations and studies were initiated after the Kobe EQ in different countries, especially in Japan in collaboration with two Japanese frontier projects engaged in seismo-electromagnetic studies during the years of 1996-2001 which contributed very much to the progress of precursor studies. Electromagnetic phenomena in possible association with EQs have been reviewed with special reference to those achievements made during and after the frontier projects. Being stimulated by the success of these Japanese frontier projects, national precursors studies devoted to EQ prediction have been developed in different countries. The most important findings during the past two decades are that many of short-term EQ precursors are non-seismic (electromagnetic, geochemical) rather than seismological, and that the ionosphere (both the lower and upper layers) and atmosphere are perturbed prior to an EQ, being coupled to the lithosphere (the presence of lithosphere-atmosphere-ionosphere coupling). As recent research activities, we will present the precursors for the recent 2011 Tohoku mega-EQ, not only electromagnetic but also of ground movements. Finally, future directions of EQ precursor studies and short-term EQ prediction will be extensively discussed with paying particular attention to the importance of multidisciplinary and multi-parameters observations and critical analyses.
\end{abstract}

\section{Introduction}

Due to enormous advance in seismology, geodesy, and so on, we find significant developments both in the long- (with the time scale of hundred years) and medium-term (with the time scale of a few decades to 
a few years) predictions of earthquakes (EQs) [Uyeda and Nagao, 2018]. On the other hand, even though short-term EQ prediction with the time scale of a month to a few days, is recognized as being most important for human beings, it is far from realization [Molchanov and Hayakawa, 2008; Hayakawa, 2015; Ouzounov et al. (Eds), 2018]. In Japan, most resources of national projects were spent on improvements of different observational networks (seismometers, crustal movements, GPS (Global positioning satellite), etc.) for monitoring the mechanical changes. Despite these improvements, seismologists could not identify any reliable EQ precursors, and they maintained a negative attitude to short-term EQ prediction, nearly the same situation in all countries.

Throughout this long period of general pessimism, however, some fervent scientists in many countries continued their positive efforts. Needless to say, short-term EQ prediction absolutely needs precursors. There have been a lot of reports of many kinds of EQ precursors (including medium- and short-term) from ancient Greek time until today [Rikitale, 2001]. As is detailed in Rikitake (2001) they can be geodetic signals such as tilt, hydrological data like level, temperature, and chemistry of underground water, electromagnetic fluctuations in various frequencies, emission of radon and other gases, and even anomalous animal behavior. Seismological events like foreshocks and preseismic quiescence in the field of seismology can also be precursors. The earlier electromagnetic findings before the 1995 Kobe EQ are very few, and there are the following outstanding two: (1) electromagnetic radiation in LF band [Gokhberg et al., 1982] and (2) DC seismic electric signals (SES) [Varotsos and Alexopoulos, 1984]. Then we believe the 1995 Kobe EQ with magnitude of 7.3 as our first milestone in the sense that we could detect a lot of electromagnetic precursors [Nagao et al., 2002], and such electromagnetic measurements started extensively all over the globe after the Kobe EQ, in particular in Japan in collaboration with the two Japanese frontier projects. It is then proven during the last few decades that the majority of the reported reliable EQ precursors are electromagnetic and that some precursory anomalies can appear in the atmosphere and even higher ionosphere (suggesting the lithosphere-atmosphereionosphere coupling (LAIC)) [Hayakawa and Molchanov (Eds), 2002; Pulinets and Boyarchuck, 2004; Hayakawa (Ed), 2009, 2012, 2013; Molchanov and Hayakawa, 2008; Hayakawa, 2015]. Our recent milestone is the publication of a monograph from American Geophysical Union (AGU) in 2018, which has recognized our seismo-electromagnetics finally as a promising new science field [Ouzounov et al. (Eds), 2018]. These observations are still going on in many countries with the final goal of short-term EQ prediction. 
This review is organized as follows. Section 2 deals with the history (or achievements) of electromagnetic phenomena associated with EQs during and after the Japanese frontier projects in order to show different precursors not only in the lithosphere, but also in the atmosphere and ionosphere. Then Section 3 presents the different precursors for the recent 2011 Tohoku EQ (not only electromagnetic phenomena, but also land movements) as recent activities. In Section 4 we provide the future directions on seismoelectromagnetics with several suggestions in the context of short-time EQ prediction, with special emphasis on the multidisciplinary and multi-parameters observations and critical analyses. Section 5 is the conclusion.

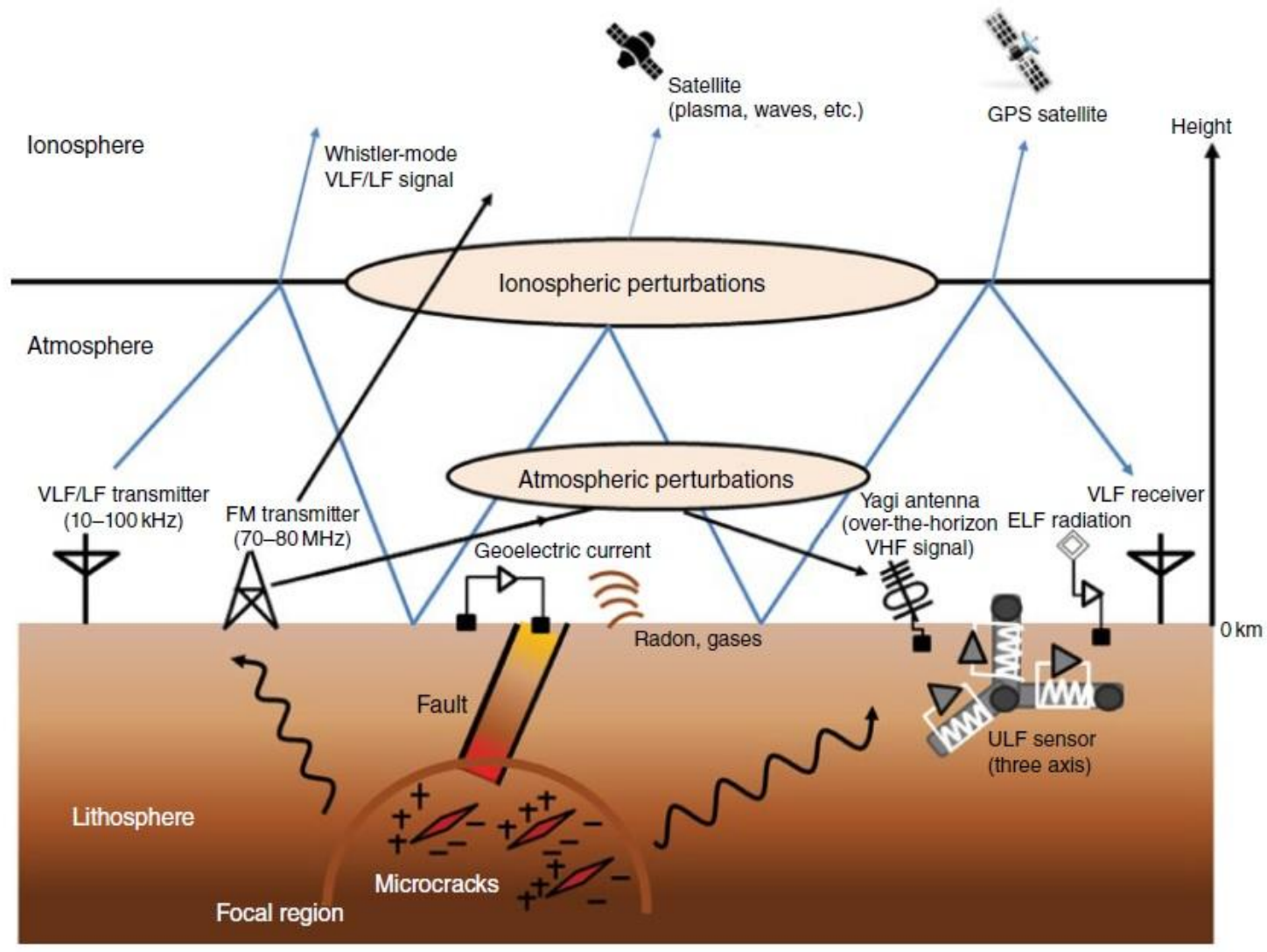

Figure 1. General view of electromagnetic phenomena in possible association with EQs and a variety of radio techniques to measure those electromagnetic effects.Adapted from Hayakawa (2018). 


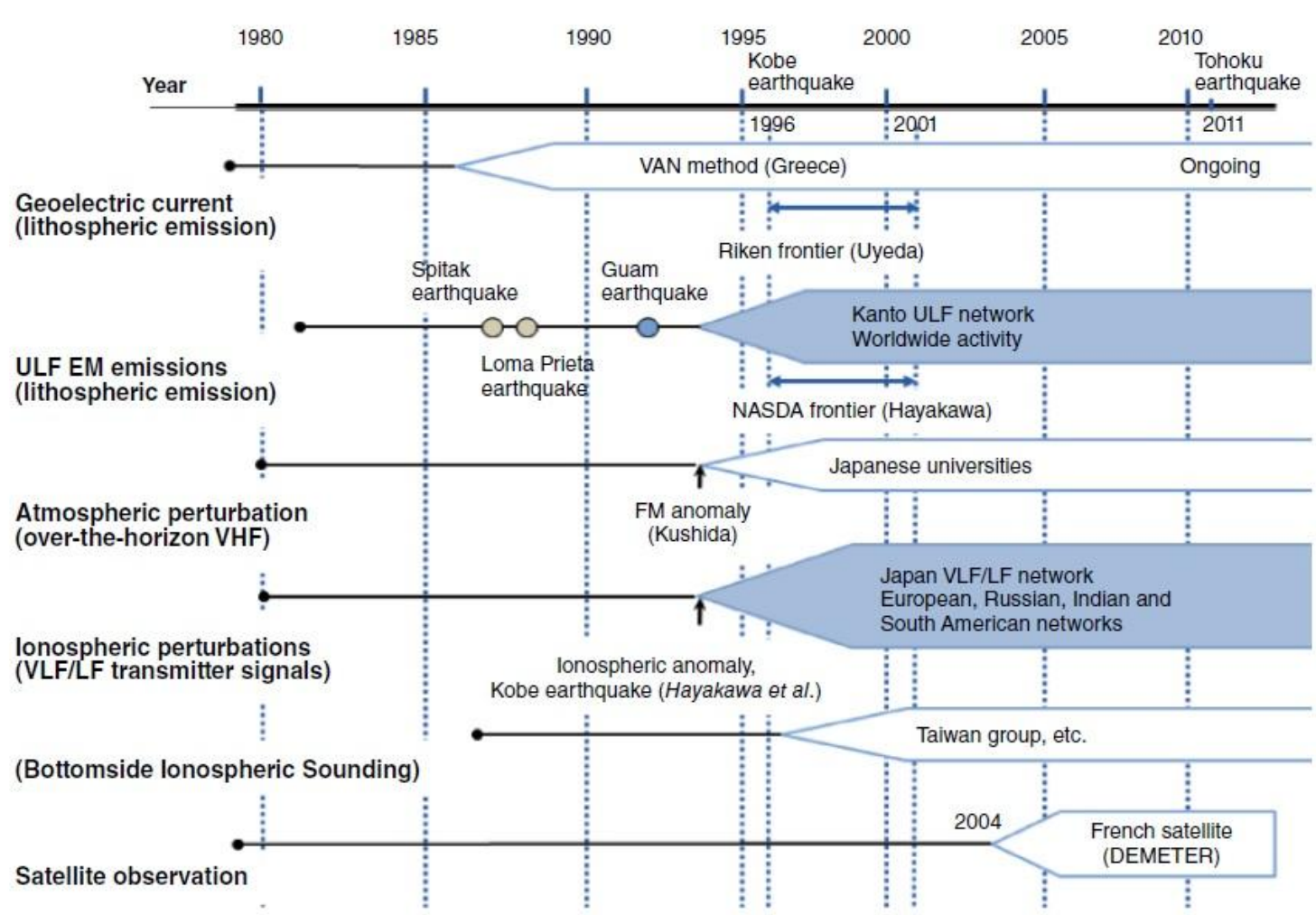

Figure 2. History of seismo-electromagnetic studies (including lithospheric effects, atmospheric effect, and ionospheric signatures). Adapted from Hayakawa (2018).

\section{Short-term EQ prediction: History}

Figure 1 illustrates a conceptual picture of seismo-electromagnetic phenomena measured by different radio techniques, while Figure 2 is a summary of the history of seismo-electromagnetic studies performed all over the world during the last few decades. The first two columns in Figure 2 refer to the lithospheric effects due to the pre-EQ microfracturing (generation of cracks) process. The third column is the seismoatmospheric effect or the phenomena taking place in the atmosphere prior to an EQ, and the last three columns all refer to the precursory ionospheric perturbations both in the lower and in the upper parts as the consequence of LAIC process [e.g., see Hayakawa (Ed), 2009]. Based on his choice (taste), the author has limited only to some outstanding observational items in Figure 2.

The first two lithospheric effects are very easy to imagine because they are the direct consequence of lithospheric pre-EQ fracture effects. That is, a DC current source is regarded as being generated in and around the EQ hypocenter during the pre-EQ preparation phase. On the other hand, radio emissions can 
appear in a wide frequency range as the effect of many oscillating charges (as currents or antennas) in the AC case, and especially ULF (ultra low frequency, frequency less than $1 \mathrm{~Hz}$ ) emission is one of the most promising candidates for EQ prediction. Other seismo-atmospheric and seismo-ionospheric effects (or perturbations occurring in the atmosphere and ionosphere) before an EQ are much more complicated to imagine than the former two items, because these are indirect effects in the sense that there appear some perturbations either in the atmosphere or in the ionosphere as the consequence of LAIC process due to the same pre-EQ effect [e.g., see Molchanov and Hayakawa (2008)]. The first geoelectric DC current measurement has the longest history as already mentioned, and the Greek group has accumulated a lot of achievements (not only case studies but also statistical studies) [so-called VAN method, Varotsos, 2015]. The second, ULF electromagnetic emissions started with the Spitak and Loma Prieta EQs [Kopytenko et al., 1990; Fraser-Smith et al., 1990], and they are likely to be of extreme importance in short-term EQ prediction studies and currently their observations are carried out in many countries [Hayakawa, 2015]. However, the number of ULF events it still not so sufficient because of its local nature, but recently Hattori (2013) has presented a statistical correlation with EQs based on a long-term (10 years) data at Kakioka, Japan. Radio emissions in other higher frequency ranges from ELF, VLF/LF, to HF or even higher, have been thoroughly reviewed in a recent monograph by Hayakawa (2015).

Seismo-atmospheric perturbation, the third item in Figure 2, was discovered for the 1995 Kobe EQ [Kushida and Kushida, 2002], which is thought as being seismo-atmospheric effect as seen from the anomalies in VHF transmitter signals and has been studied initially in Japan but recently in different countries as well [Fukumoto et al., 2011; Fujiwara et al., 2004; Devi et al., 2012]. Further, many types of infrared (IR) parameters in the atmosphere have been recognized as useful possible EQ anomalies [e.g., Tronin et al., 2002]. Among them, most cited recently are the brightness temperature, outgoing longwave radiation (OLR), surface latent heat flux, but this atmospheric topic is still controversial as an EQ precursor [Ouzounov et al., 2011]. In contrast with this, a much more convincing phenomenon in the atmosphere has been found with statistical significance by Molchanov et al. (2013) and Schekotov et al. (2007), and this ULF/ELF (frequency=1-10 Hz) impulsive radiation has been suggested as the most attractive signature of seism-atmospheric effects [Schekotov et al., 2013; Schekotov and Hayakawa, 2017].

The last item of ionospheric perturbation has a relatively short history. Since the convincing evidence of ionospheric perturbation for the 1995 Kobe EQ was found with the use of subinospheric VLF 
propagation [Hayakawa et al., 1996], this has led to the widespread use of VLF/LF network all over the world, including Europe, Russia, India, and South America [Biagi et al., 2011; Rozhnoi et al., 2004; Chakrabarti et al., 2010]. In parallel with the lower ionospheric studies, a lot of attention has been paid to the upper ionosphere like F region [Pulinets and Boyarchuk, 2004] because there are available a lot of ionosonde (foF2) observations in the world and also the GPS TEC (Total electron content) data have been available recently. There have been, then, established statistical correlations of the ionospheric perturbations (not only in the lowest ionosphere, but also the upper F region ionosphere) with EQs [Liu et al., 2006; Liu, 2009; Hayakawa et al., 2010; Sorokin et al., 2015], which means that the ionosphere as the upper layer is definitely perturbed indicating the LAIC process, even though the mechanism needs further studies as current interest.

The French satellite DEMETER, dedicated to the study of seismo-electromagnetics, was launched in 2004, and a lot of scientific success has been achieved on the study of how the ionosphere is disturbed due to the lithospheric pre-EQ effect or LAIC process [Parrot, 2013; Parrot and Li, 2018]. As you may know, China succeeded in launching the next satellite, CSES in 2018 and we will expect a lot of new findings from this satellite.

One more important point as related to Figure 2 is that we, the seismo-electromagnetic group in Japan, were funded only once just after the 1995 Kobe EQ during the years of 1996-2001. Two institutions were asked to do a feasibility study of electromagnetic effects in short-term EQ prediction during 5 years (1996-2001): (i) Institute of Physical and Chemical Research (RIKEN) and (ii) former National Space Development Agency of Japan (NASDA, presently JAXA) in the framework of Earthquake Integrated Frontier by the former Science and Technology Agency (now Mext). The RIKEN frontier project was led by Prof. S. Uyeda and the latter, NASDA's frontier project, by the author of this review. The RIKEN group had a lot of interests in installing geoelectric SES measurements in Japan, together with the measurement of ULF electromagnetic emissions, while we, the NASDA team, tried to expand the observation area as much as possible by including the ULF electromagnetic waves in the lithosphere, different atmospheric effects, and also ionospheric signatures with ground- and satellite-based observations. Of course, our main concern has been the use of subionospheric VLF/LF signals. The success of these Japanese frontier projects has stimulated a lot of interests in other countries including Taiwan, India, Mexico, EU, China etc. 


\section{Recent findings for the 2011 Tohoku EQ}

How about the precursors for the 2011 Tohoku mega-EQ? There were in fact precursors, although most of them were only recognized afterward. There are also encouraging signs for future developments in short-term prediction. This part is based on Hayakawa (2018), mainly referring to the results by Japanese colleagues. However, we have also cited the corresponding some precursors by foreign scientists with satellite observations (some of them are shown in the book by [Ouzounov et al. (Eds), 2018].

\subsection{Electromagnetic phenomena}

\section{(a) Lithospheric effects.}

Kopytenko et al. (2012) investigated the magnetic field variations at three observatories (Esashi, Mizusawa, and Kakioka) over the 11-year period of 1 January 2000 through 31 January 2011. They found a medium-term anomaly around 3 years before the EQ, and also a short-term precursor in the ULF frequency range of $0.0033-0.01 \mathrm{~Hz}$ was observed starting 22 February 2011. Another type of anomalous daily variation of magnetic data was observed approximately 2 months prior to the EQ (Xu et al., 2013). Hayakawa et al. (2015) performed a critical, so-called natural time (NT) analysis of the ULF data for this EQ, and found that the horizontal magnetic field component $(\mathrm{f}=0.03-0.05 \mathrm{~Hz}$ ) fulfilled all criticality conditions for 3-5 March, about a week before the EQ, which is a definite short-term precursor.

\section{(b) Atmospheric effects.}

Ohta et al. (2013) observed ULF/ELF atmospheric radiation on the basis of measurements at three stations in the Nagoya area: Nakatsugawa, Shinojima and Izu. It was found that ULF/ELF impulsive radiation was reliably recorded on 6 March as a short-term precursor. Further confirmation on its seismic origin was provided by the observational fact that the azimuths of the radiation source from all stations coincided approximately with the region of the forthcoming EQ. Schekotov and Hayakawa (2015) investigated the 5-year ULF data at Kakioka around the EQ, who have found the ULF (10 to $100 \mathrm{mHz}$ ) radiation in the vertical magnetic field component which is correlated with seismicity.

Ouzounov et al. (2011) investigated the multi-parameters analysis for this EQ, and found that OLR exhibited anomalies on 21-24 February and also 7-11 March. 


\section{(c) Ionospheric signatures}

Hayakawa et al. (2012, 2013a, b) studied the data from their VLF/LF network observation and found a very convincing short-term precursor to this EQ. A remarkable anomaly characterized by a decrease in the nighttime amplitude and an enhancement in amplitude fluctuation was detected on 5 and 6 March on the propagation path from the NLK (Jim Creek, USA) transmitter to Chofu (together with similar signatures at other stations of Kasugai (Nagoya) and Kochi).

Schekotov et al. (2013) and Hayakawa et al. (2013c) studied the depression of ULF horizontal magnetic field variations of magnetospheric origin, which is not so popular in the scientific society but is very sensitive to the pre-EQ effect just seeing the same lower ionospheric perturbation as seen by subionospheric VLF/LF propagation (Schekotov and Hayakawa, 2017). An anomaly in ULF depression is found on 6 March, which is consistent with the corresponding VLF result.

Another issue of current debate is the imminent precursor to this EQ, which is found from the GPS TEC data and which happens about 40 hours before the EQ (Heki, 2011; Kamogawa and Kakinami, 2013), but further study is required (Iwata and Umeno, 2016).

\subsection{Land movements}

Another highly promising development is the detection of preseismic land movements using GPS data [e.g. Chen et al., 2013], Kamiyama et al. (2014) investigated GPS data on land movements extensively for a period of about 10 years before the $2011 \mathrm{EQ}$. Their clearest anomaly in crustal movements is the short-term precursor before the EQ. The longitudinal movement change exhibited a sharp increase over 610 March, and stayed flat, in readiness to rebound following the activity of 3-6 March. This kind of information on crustal movements should be combined with electromagnetic signatures for further understanding of the mechanism of seismo-electromagnetic phenomena.

\subsection{Overall summary of precursors}

Summarizing the above various kinds of precursors, it is found that there are two main periods of anomalies. The first is 22-24 February and 3-7 March, a few days to about a week before the EQ. A combination or coordination of these data with particular attention to their temporal and spatial evolutions 
will help us to better understand the LAIC mechanism (to be discussed later) for this EQ (that is, which mechanism is working for this particular EQ?).

\section{Future prospects}

The most important steps of short-term EQ prediction can be summarized as follows (Molchanov and Hayakawa, 2008; De Santos et al., 2019).

(1) Present a physical model which can explain the proposed precursor anomaly.

(2) Define exactly an anomaly and describe how it can be observed.

(3) Explain how the precursor information can be translated into an EQ forecast and specify such a forecast in terms of probabilities for given time/space/magnitude windows.

(4) Perform a test over some time that allows us to evaluate the proposed precursor and its forecasting power.

(5) Report on successful prediction, missed EQs, and false predictions.

Theoretically speaking, all of these points should be sequential; that is, any precursor must satisfy all of them. However, the author thinks that the first point (1) of establishing a physical model is too difficult to reach for any one of the proposed precursors, and even more difficult is the elucidation of the LAIC process. So, the author thinks that finding any precursor and estimating its forecast power of the above points of (2)-(5) should be extensively studied, and eventually these points have been currently investigated in the world, and we leave the most tough point (1) aside though a lot of efforts should be performed.

When thinking of the above points (2)-(5), we normally use a probabilistic terminology to estimate the validity of any proposed precursor or precursor efficiency, and we follow the concept of Console (2001). We use "success rate", false alarm rate, failure rate, and "probability gain (PG)". The last is the most important parameter on how good any particular anomaly can be used in the EQ prediction. When the PG value is greater than unity, a method of EQ prediction with this anomaly is considered significant. Even though the book by Molchanov and Hayakawa (2008) was written about 10 years ago, they showed the estimations of PGs for nearly all kinds of reported precursors as described already here in this review (short-term and imminent precursors) based on the relatively long-term observations performed until that 
time. It is found from their tables that especially the ionospheric precursors as based on VLF/LF sounding, attained the highest value of $P G=3-5$, indicating the lowest ionosphere as a promising precursor. Recently there have been published papers on the more modern statistical studies with the use of ECA (event coincidence analysis), ROC (Receiver Operating Characteristics) analysis and others, as applied to seismicity, SES signals, ULF emissions etc. [Sarlis et al., 2010; Sarlis, 2019], and with Molchan error diagram as applied to ULF radiation [Hattori and Han, 2018].

As concerns with items (4) and (5), we have to tell the readers the moment of truth. To the author's knowledge, there are already three groups launching the prospective EQ forecasts using studied precursors. The first is by the Greek group (led by Prof. Varotsos) with the use of SES observation, the second is our Hi-SEM group with the use of a network observation of subionospheric VLF/LF propagation signals. And the last is by a Russian group (led by Dr. Schekotov of Institute of Physics of the Earth) in cooperation with the group in Kamchatka, on the basis of observation of seismo-atmospheric ULF/ELF radiation. These three observational phenomena are accidentally lithospheric, ionospheric and atmospheric effects respectively, which are suggested as the most promising candidate for EQ prediction as seen from this review.

Readers of this review could also understand that various radio techniques were used in this short-term EQ prediction as in Figure 1. These are, in principle, two possible ways of observing different seismogenic effects: passive and active methods. As for the first passive method, we try to detect any electromagnetic radiation directly from the lithosphere in a wide frequency range (from DC/ULF to VHF or even higher), and high-sensitive detectors (receivers) are required to detect those seismogenic emissions with relatively weak intensity. The possible detection range of electromagnetic radiation seems to depend on frequency. For example, the detection range for seismogenic ULF emissions is on the order of $100 \mathrm{~km}$, this kind of local measurement will, in turn, enable us to pinpoint an emission source using direction finding techniques [Kopytenko et al., 2006]. But, the detection range becomes larger on the order of $1000 \mathrm{~km}$ at ELF and VLF, but an alternative problem will arise on how to locate the emission source, and so we will need to adopt a very sophisticated array system, such as multi-point direction finding systems [Schekotov and Hayakawa, 2017]. Second way of observing seismogenic effects is a socalled active probing method, in which signals from any transmitters at various frequencies such as at VLF/LF and VHF, are utilized to study perturbations occurring in the atmosphere or in the ionosphere in possible association with pre-EQs effects [Molchanov and Hayakawa, 2008]. The most important 
advantage of this integrated measurement is that any EQs occurring just around the propagation path between the receiver and transmitter give rise to a significant anomaly in the propagation characteristics at the receiver, making it possible for us to accumulate the number of events very easily, just as cases of subionospheric VLF/LF [Hayakawa et al., 2010; Chakrabarti (Ed), 2010] and over-the-horizon VHF propagations [Kushida and Kushida, 2002]. So these integrated measurements will enable us to study extensively the points (2)-(5) in the EQ prediction. Being closely associated with the achievements of above points (2)-(5), we address the technical problems below.

\subsection{Long-period ground-based observations}

Numerous electromagnetic precursors using radio techniques have been found, but the most important point is that we must carry out long-period (at least 5 years, or preferably 10 years or so) ground-based observations (preferably with your own measurement) in order to establish a clear statistical correlation between any precursor and EQs [as done in Liu (2009), Hayakawa et al. (2010), Hattori (2013)]. We know that the most serious problem in continuous observations is the presence of data gaps due to malfunction of equipment and industrial interferences, so special care should be paid on the importance of continuous observations of any observational item. This long-term observation will be of fundamental importance in studying the points (2)-(5) in the EQ forecast. Furthermore, only from this kind of longterm continuous observations, there exists a high possibilty for us to discover a new phenomenon in seismogenic studies. For example, anomalies in Schumann resonances (SR) were our discovery [Hayakawa et al., 2005], but we show an additional latest finding also in the ELF band: SR-like unusual line emissions observed in possible association with EQs [Ohta et al., 2009; Hayakawa et al., 2011], which can be interpreted in terms of excitation of gyrotropic waves in a thin layer of the lower ionosphere [Sorokin and Hayakawa, 2008]. This kind of discoveries will follow in future as well.

\subsection{Development of highly-sensitive equipment}

In any frequency range as discussed in this review, detectors for seismogenic electromagnetic signatures are the first step to distinguish weak seismogenic effects from other interference noise. So we have to develop, initially, highly-sensitive detectors in any frequency bands, for example, a new DC geopotential detector free from the problem of contact potential with the ground, a new type of ULF sensors (with new 
principles), various kinds of direction finding systems in ULF, ELF/VLF,..., VHF or so, VLF/LF Doppler-shift observation [Asai et al., 2011], and VHF interferometer direction finding [Yasuda et al., 2009]; hence, we strongly invite radio scientists and engineers to pursue their EQ prediction research in this direction. Development of such sensitive receivers will surely provide us with finding (or discovery) of new seismogenic effects.

\subsection{Satellite observations}

The above technical problems are associated with ground-based observations of different electromagnetic (including geochemical) phenomena, but we have to emphasize the important role of satellite observation. We are happy to understand that the observation by DEMETER launched in 2004 has yielded a lot of scientific outputs on ionospheric plasma perturbations, electromagnetic waves, particles etc [Parrot, 2013; Parrot and Li, 2018]. The Chinese satellite dedicated to seismo-electromagnetics, next to Demeter, has been successfully launched in 2018, which will surely trigger again much international collaboration. We, Japanese, have a strong desire to have such a satellite. Furthermore, the data available from other satellites have been utilized to study the in-situ magnetic field, plasma density etc. and to obtain surface information (such as OLR) [Tronin et al., 2002; Ouzounov et al., 2018; Tramutoli et al., 2018].

\subsection{Signal processing techniques}

As related with the development of new measurement techniques, another essential problem arises in developing new signal processing techniques on how to extract anomalies from long-term data. Even in the field of DC/ULF and VLF/LF, you can understand how important new signal processing techniques were in the study of EQ prediction. Much progress has been achieved in this vital field of signal processing techniques, including principal component analysis (independent component analysis) [Gotoh et al., 2002], fractal analysis to study a nonlinear process in the lithosphere [Hayakawa et al., 1999; Smirnova et al., 2014; Ida et al., 2006, 2012] and various kinds of direction finding [e.g., Kopytenko et al., 2006]. Next, we try to think of possible signal processing in our VLF studies. As an example of application of our subionospheric VLF/LF observation to real EQ prediction, we have to determine three parameters of an EQ (when, where, and how big (magnitude)). In order to increase the prediction accuracy, one way is definitely to increase the number of receiving VLF/ LF stations (we are just now 
doing) and another way is to make full use of multi-propagation path data with a new concept of signal processing technique such as AI (artificial intelligence) [Potirakis et al., 2018c].

The conventional signal processing is based on finding statistically any extreme values in the time series data, even though some of them are very modern. However, the occurrence of an EQ can be considered physically as a self-organized criticality phenomenon, or a phase transition. This kind of new concepts will enable us to confirm whether this anomaly is not attributed to any other agents, but to the criticality due to the pre-EQ fracturing process. Fractal analysis is one of those critical analyses, but a new conceptual analysis method has been proposed by Varotsos (2005) named natural time (NT); for example, in their NT analysis the variance (к1) is taken as an order parameter. This NT analysis has been often applied to SES signals, seismicity etc. [Varotsos, 2005; Sarlis et al., 2010; Sarlis, 2019]. Despite that we have done a lot of fractal analysis (both mono- and multi-fractal) in ULF emission data [Ida et al., 2006, 2012], we have tried to apply this NT method to other seismogenic phenomena including ULF emissions, VLF/LF propagation data etc. This NT analysis is found to be very suitable for point processes like SES and seismicity, but we have found that extensive studies are required on which kind of parameters should be appropriate for NT application for continuous processes. But if we can find some appropriate parameters, we could have a lot of successes in finding criticality even for continuos processes like ULF emissions, VLF propagation [Potirakis et al., 2016, 2018a, b, 2019; Contoyiannis et al., 2016]. In future we cannot predict an EQ without the help of these critical analyses.

In addition to the above technical problems related to radio engineering, the final goal of our seismoelectromagnetic study is to have better understanding of physical mechanisms of different kinds of seismogenic effects (seismogenic emissions, seismo-atomoshperic and ionospheric perturbations, and plasma and electromagnetic signatures in the ionosphere), and it is obvious that elucidation of those physical mechanisms will help much in the improvement of practical EQ prediction as well. These difficult, but challenging, problems to be solved in future are summarized as follows:

\section{4.a Multi-parameter observations}

As was seen from this review, you can understand that already a few physical models for LAIC process have been proposed as in Figure 3: Chemical channel, electromagnetic channel, electrostatic channel, and atmospheric oscillation channel. In the chemical channel, radon emanation is a main player, leading to the 
ionization in the atmosphere, generation of electric field, and leading to the ionospheric perturbation [Pulinets, 2009; Sorokin and Hayakawa, 2014]. In the electrostatic hypothesis (not shown in Figure 3) the positive hole carriers appear on the Earth's surface due to the stress-induced effect [Freund, 2009]. The last atmospheric oscillation hypothesis is that acoustic waves (AWs) or atmospheric gravity waves (AGWs) are excited by the ground motion due to the pre-EQ crustal resonance [Molchanov and Hayakawa, 2008; Hayakawa et al., 2018]. However, it is clear that those hypotheses should be verified experimentally with the integration of observations in different regions of lithosphere, atmosphere, and ionosphere. One possible way of this kind of coordination of various observational items is to establish a field test site (such as Kamchatka as a part of the collaboration between Russians and Japanese [Uyeda et al., 2002]). The primary requirement for such a test field site is that it must be located at a place free from man-made interference noise. The second requirement will be that we have to observe simultaneously as many phenomena as possible, as in Figure 2, in order to achieve extensive coordination of observational data, which will enable us to observationally verify any particular hypothesis. A combination of phenomena taking place simultaneously in the lithosphere, atmosphere and ionosphere, is highly required with some examples as done in [Pulinets and Boyarchuk 2004, Ouzounov et al. 2018 and Yang et al. 2019]. Finally we have to mention at the moment that any of these hypotheses has not yet been verified observationally to our satisfaction, and a lot of works remain in front of us.

Another new direction in the LAIC studies is the integrated multiparameter use of ground- and satellite-based data. Recently a paper has been published by [Yang et al. 2019], who have shown the presence of abnormal AGW wave activity in the atmosphere (stratosphere) in the height range of 10-50 $\mathrm{km}$ with the use of ERA data and found that those stratospheric AGW activity is consistent (both in time and space) with the corresponding lower ionospheric perturbation as seen by subionospheric VLF data. This is just a case study for the 2016 Kumamoto EQs, but this is the first convincing evidence to the atmospheric oscillation channel of the LAIC process. These directions are highly recommended in order to obtain the direct evidence in favor of any hypothesis. 


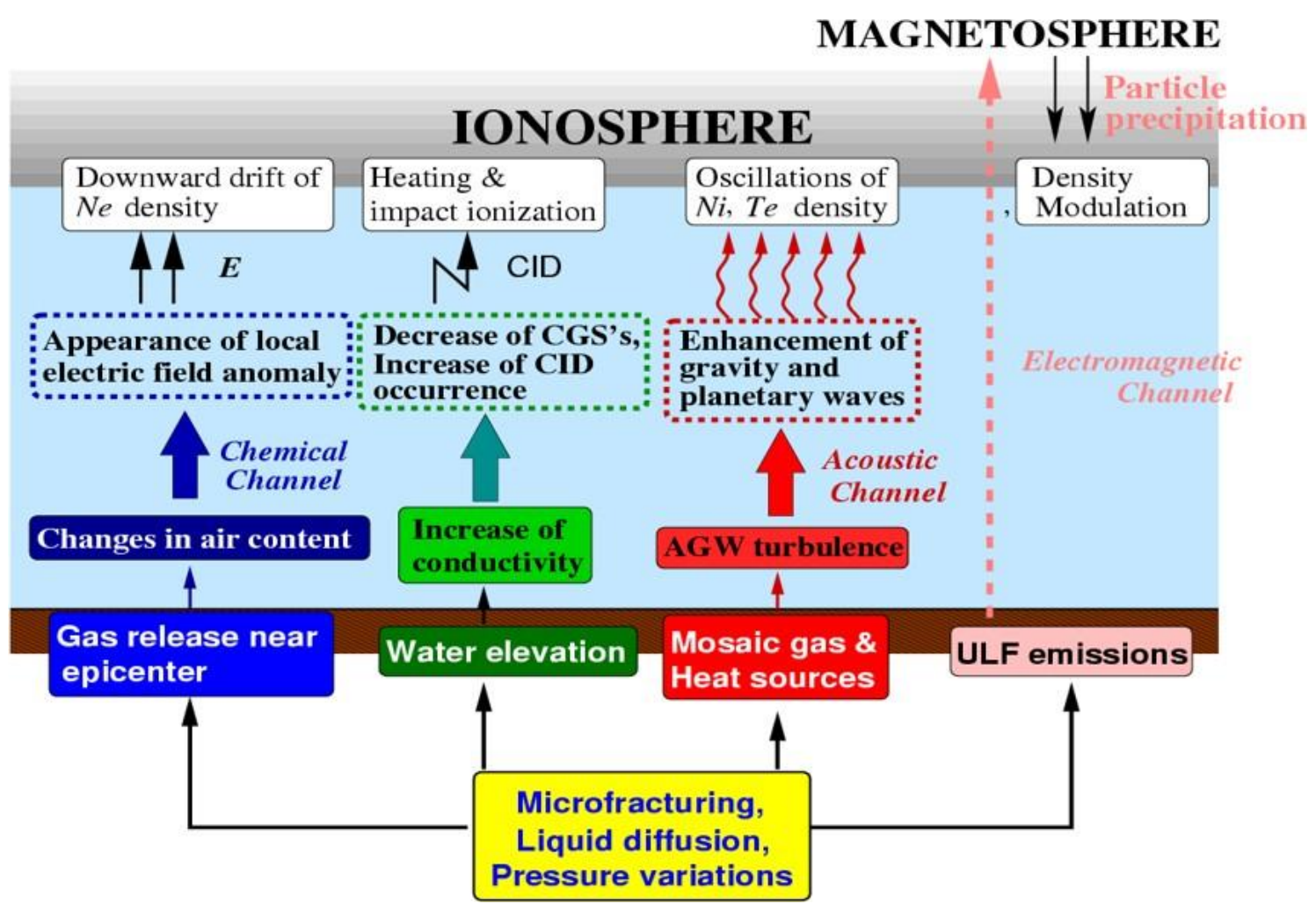

Figure. 3 Schematic illustration of the lithosphere-atmosphere-ionosphere coupling (LAIC): ( i ) chemical (+electric field) channel, ( ii ) atmospheric oscillation channel, and (iii) electromagnetic channel. Reproduced with permission from Hayakawa (Ed) (2009). () 2009, Transworld Research Network, Trivandrum, India.

\section{4.b Studies on the mechanisms of generation of seismogenic emissions}

Recently a lot of attention has been paid to the elucidation of LAIC process, but it seems that less attention is paid to the generation mechanisms of each electromagnetic phenomenon, for example, the generation of DC SES signals [Varotsos, 2005], ULF emissions (Molchanov and Hayakawa, 2008), and electromagnetic radiation in higher frequency ranges (ELF, VLF/LF, HF or so). Furthermore, the generation of a recent phenomenon such as seismo-atmospheric ULF/ELF emissions is extremely poorly understood, even though it might be atmospheric discharges [Schekotov and Hayakawa, 2017]. Efforts to those studies on the generation mechanism of each phenomenon should be performed within the context of LAIC process, together with the information of pre-EQ fracturing in the lithosphere as the primary agent. 


\section{Conclusion}

This review has introduced the concept of seismo-electromagnetics for short-term EQ prediction. Our fundamental spirit of these studies is to use some universal tools to look at our Earth system of a complex coupled system of lithosphere, atmosphere, and the ionosphere with the multidisciplinary and multiparameter observations. Another important frame is that of dynamical systems approaching a critical point when the macroscopic properties of the system change drastically during the pre-EQ preparation phase. Here we have to mention that being closely related with criticality, there have been going on the works on triggering processes of EQs [Adushkin and Kocharyan, 2011].

The author is very optimistic about the future of short-term EQ prediction. An extensive statistical big data analysis would be, even in future, important to confirm or confute the individual case studies (though no definite conclusions can be arisen, because high correlation does not always mean causation); but can be of great help in proposing a physical framework of the chain of processes that could occur before a large EQ.

Finally the author wants to invite many young scientists and engineers to join our new and challenging science field with preferably your own measurement, but even if it is difficult, with the retrospective analysis of any available data from ground-based observations such as ionosonde (foF2) and GPS TEC data. Also, different satellite data are available nowadays, which will be useful in your seismoelectromagnetics studies. A lot of efforts will be required in order to have a bright future for short-term EQ prediction, but we can dream that short-term EQ prediction will be realized in near future.

\section{Reference}

Adushkin, V. V., and Kocharyan, G. G., Trigger processes in geosystems, Izvestia, Physics of the Solid Earth, 47, No. 3, 259-261, 2011.

Asai G, S., Yamamoto, S. Kasahara, Y., Hobara, Y., Inaba, T., and Hayakawa, M., Measurement of Doppler shifts of short-distance subionospheric LF transmitter signals and seismic effects, J. Geophys. Res., doi:10.1029/2010JA016055, 2011.

Biagi, P. F., T. Maggipinto, F. Righetti, D. Loiacono, et al., The European VLF/LF radio network to search for earthquake precursors: Setting up and natural/man-made disturbances, Natural Hazards Earth System Sci., 11(2), 333-341, doi:10.5194/nhess-11-333-2011, 2011 
Chakrabarti, S. K. (Ed), Propagation Effects of Very Low Frequency Radio Waves, American Institute of Physics, vol. 1286, 362p, 2010.

Chen, C. H., S. Wen, J. Y. Liu, et al. (2013), Surface displacements in Japan before the 11 March 2011 M9.0 Tohoku-oki earthquake, J. Asian Earth Sci., 80, 165-171.

Console, R., Testing earthquake forecast hypotheses, Tectonophysics, 338, 261-268, 2002.

Contoyiannis, Y., S. M. Potirakis, K. Eftaxias, M. Hayakawa and A. Schekotov, Intermittent criticality revealed in ULF magnetic fields prior to the 11 March 2011 Tohoku earthquake $(\mathrm{Mw}=9)$, Physica A, http://dx.doi.org/10.1016/j.physa.2016.01.065, 2016.

De Santos, A., C. Abbattisa, L. Alfonsi, L. Amoruso, S. A. Campuzano, M. Carbone, C. Cesaroni, G. Cianchini, et al., Geosystematic view of earthquakes, Entropy, 21, 412, doi:10.3390/e21040412, 2019.

Devi, M., A. K. Barbara, Ya. Yu. Ruzhin, and M. Hayakawa, Over-the-horizon anomalous VHF propagation and earthquake precursors, Survey Geophys., 33, 1081-1106, doi:10.1007/s10712-012-9185z, 2012.

Fraser-Smith, A. C., A. Bernardi, P. R. McGill, M. E. Ladd, R. A. Helliwell, and O. G. Villard Jr, Lowfrequency magnetic field measurements near the epicenter of the Ms 7.1 Loma Prieta earthquake, Geophys. Res. Lett., 17, 1465-1468, 1990.

Freund, F., Stress-activated positive hole charge carriers in rocks and the generation of pre-earthquake signals, in "Electromagnetic Phenomena Associated with Earthquakes", Ed. by M. Hayakawa, Transworld Research Network, Trivandrum India, 41-96, 2009.

Fujiwara, H., M. Kamogawa, M. Ikeda, J. Y. Liu, H. Sakata, Y. I. Chen, H. Ofuruton, S. Muramatsu, Y. J. Chuo, and Y. Ohtsuki, Atmospheric anomalies observed during earthquake occurrences, Geophys. Res. Lett., 31, L17110, 2004.

Fukumoto, Y., M. Hayakawa, and H. Yasuda, Investigation of over-horizon VHF signals associated with earthquakes, Natural Hazards Earth System Sci., 1, 107-112, 2001.

Gokhberg, M. B., V. A. Morgounov, T. Yoshino, and I. Tomizawa, Experimental measurement of electromagnetic emissions possibly related to earthquakes in Japan, J. Geophys. Res., 87, 7824-7828, 1982.

Gotoh, K., Y. Akinaga, M. Hayakawa, and K. Hattori, Principal component analysis of ULF geomagnetic data for Izu islands earthquakes in July 2000, J. Atmos. Electr., 22, 1-22, 2002.

Hattori, K., ULF geomagnetic changes associated with major earthquakes, in "Earthquake Prediction Studies: Seismo Electromagnetics", Ed. by M. Hayakawa, TERRAPUB, Tokyo, 129-152, 2013. 
Hattori, K., and P. Han, Statistical analysis and assessment of ultralow frequency magnetic signals in Japan as potential earthquake precursors, in "Pre-earthquake Processes: A multidisciplinary approach to earthquake prediction studies", Ed. by D. Ouzounov et al., AGU Geophysical Monograph 234, Wiley, 229-240, 2018.

Hayakawa, M. (Ed), Electromagnetic Phenomena Associated with Earthquakes, Transworld Research Network, Trivandrum India, 279p, 2009.

Hayakawa, M. (Ed), The Frontier of Earthquake Prediction Studies, Nihon-Senmontosho-Shuppan, Tokyo, 794p, 2012.

Hayakawa, M. (Ed), Earthquake Prediction Studies: Seismo Electromagnetics, TERRAPUB, Tokyo, $168 \mathrm{p}, 2013$.

Hayakawa, M., Earthquake Prediction with Radio Techniques, John Wiley \& Sons, Singapore, 294p, 2015.

Hayakawa, M., Earthquake precursor studies in Japan, in "Pre-earthquake Processes: A multidisciplinary approach to earthquake prediction studies", Ed. by D. Ouzounov et al., AGU Geophysical Monograph 234, Wiley, 7-18, 2018.

Hayakawa, M., and O. A. Molchanov (Eds), Seismo Electromagnetics: Lithosphere-AtmosphereIonosphere Coupling, TERRAPUB, Tokyo, 477p, 2002.

Hayakawa, M., O. A. Molchanov, T. Ondoh, and E. Kawai, The precursory signature effect of the Kobe earthquake on VLF subionospheric signals, J. Comm. Res. Lab., Tokyo, 43, 169-180, 1996.

Hayakawa, M., T. Itoh, and N. Smirnova, Fractal analysis of ULF geomagnetic data associated with the Guam earthquake on August 8, 1993, Geophys. Res. Lett., vol. 26, No. 18, 2797-2800, 1999.

Hayakawa, M., K. Ohta, A.P. Nickolaenko, and Y. Ando, Anomalous effect in Schumann resonance phenomena observed in Japan, possibly associated with the Chi-chi earthquake in Taiwan, Ann. Geophysicae, 23, 1335-1346, 2005.

Hayakawa, M., Y. Kasahara, T. Nakamura, F. Muto, T. Horie, S. Maekawa, Y. Hobara, A. Rozhnoi, M. Solovieva, and O. A. Molchanov, A statistical study on the correlation between lower ionospheric perturbations as seen by subionospheric VLF/LF propagation and earthquakes, J. Geophys. Res., 115, A09305, doi:10.1029/2009JA015143, 2010.

Hayakawa, M., Y. Hobara, K. Ohta, J. Izutsu, A. P. Nickolaenko, and V. M. Sorokin, Seismogenic effects in the ELF Schumann resonance band, IEEJ Transactions on Fundamentals and Materials, 131(9), 684690, 2011. 
Hayakawa, M., Y. Hobara, Y. Yasuda, H. Yamaguchi, K. Ohta, J. Izutsu, and T. Nakamura, Possible precursor to the March 11, 2011, Japan earthquake: ionospheric perturbations as seen by subionospheric very low frequency/low frequency propagation, Ann. Geophysics (Italy), 55 (1), 95-99, doi: 10.4401/ag5357, 2012.

Hayakawa, M., A. Rozhnoi, M. Solovieva, Y. Hobara, K. Ohta, A. Schekotov, and E. Fedorov, The lower ionospheric perturbation as a precursor to the 11 March 2011 Japan earthquake, Geomatics, Natural Hazards, and Risk, 4(3), 275-287, doi:org/ 10.1080/19475705.2012.751938, 2013a.

Hayakawa, M., Y. Hobara, A. Rozhnoi, M. Solovieva, K. Ohta, J. Izutsu, T. Nakamura, and Y. Kasahara, The ionospheric precursor to the 2011 March 11 earthquake based upon observations obtained from the Japan-Pacific subionospheric VLF/LF network, Terr. Atmos. Ocean. Sci., 24 (3), 393-408, doi: 10.3319/TAO.2012.12.14.01(AA), 2013b.

Hayakawa, M., A. Schekotov, E. Fedorov, and Y. Hobara, On the ultra-low-frequency magnetic field depression for three huge oceanic earthquakes in Japan and in the Kurile islands, Earth Science Research, 2 (1), 33-42, 2013c.

Hayakawa, M., A. Schekotov, S. Potirakis, and K. Eftaxias, Criticality features in ULF magnetic fields prior to the 2011 Tohoku earthquake, Proc. Japan Acad., Ser. B, 91, 25-30, doi: 10.2183/pjab.91.25, 2015.

Hayakawa, M., T. Asano, A. Rozhnoi, and M. Solovieva, Very-low- and low-frequency sounding of ionospheric perturbations and possible association with earthquakes, in "Pre-Earthquake Processes: A multidisciplinary approach to earthquake prediction studies", Ed. by D. Ouzounov et al., AGU Monograph 234, Wiley, 277-304, 2018.

Heki, K., Ionospheric electron enhancement preceding the 2011 Tohoku-oki earthquake, Geophys. Res. Lett., 38, L17312, doi:10.1029/2011GL047908, 2011.

Ida, Y., and M. Hayakawa, Fractal analysis for the ULF data during the 1993 Guam earthquake to study prefracture criticality, Nonlinear Processes Geophys., 13, 409-412, 2006.

Ida, Y., D. Yang, Q. Li, H. Sun, and M. Hayakawa, Fractal analysis of ULF electromagnetic emissions in possible association with earthquakes in China, Nonlinear Processes Geophys., 19, 577-583, doi:10.5194/npg-19-577-2012, 2012.

Iwata, T., and K. Umeno, Correlation analysis of preseismic total electron content anomalies around the 2011 Tohoku earthquake, J. Geophys. Res., 121, 8969-8984, doi:10.1002/2016JA023036, 2016.

Kamiyama, M., M. Sugito, M. Kuse, A. Schekotov, and M. Hayakawa, On the precursors to the 2011 Tohoku earthquake: crustal movements and electromagnetic signatures, Geomatics, Natural Hazards, and Risk, doi:10.1080/19475705.2014.937773, 2014. 
Kamogawa, M., and Y. Kakinami, Is an ionospheric electron enhancement preceding the 2011 Tohokuoki earthquake a precursor?, J. Geophys. Res., Space Physics, 118, 1751-1754, doi:10.1002/jgra.50118, 2015.

Kopytenko, Yu. A., V. S. Ismaguilov, K. Hattori, and M. Hayakawa, Determination of heart position of a forthcoming strong EQ using gradients and phase velocities of ULF geomagnetic disturbances, Phys. Chem. Earth, 31, 292-298, 2006.

Kopytenko, Yu. A., T. G. Matiashvily, P. M. Voronov, E. A. Kopytenko, and O. A. Molchanov, Detection of ULF emission connected with the Spitak earthquake and its aftershock activity based on geomagnetic pulsations data at Dusheti and Vardzia observatories, Phys. Earth Planet. Inter., 77, 85-95, 1990.

Kopytenko, Yu. A., V. S. Ismaguilov, K. Hattori, and M. Hayakawa, Anomaly disturbances of the magnetic fields before the strong earthquake in Japan on March 11, 2011, Ann. Geophysics (Italy), 55 (1), 101-107, doi: 10.4401/ag-5260, 2012.

Kushida, Y., and R. Kushida, Possibility of earthquake forecast by radio observations in the VHF band, J. Atmos. Electr., 22, 239-255, 2002.

Liu, J. Y., Earthquake precursors observed in the ionospheric F-region, in "Electromagnetic Phenomena Associated with Earthquakes", Ed. by M. Hayakawa, Transworld Research Network, Trivandrum India, 187-204, 2009.

Liu, J. Y., Y. I. Chen, Y. J. Chuo, and C. S. Chen, A statistical investigation of pre-earthquake ionospheric anomaly, J. Geophys. Res., 111, A05304, doi:10.1029/2005JA011333, 2006.

Molchanov, O. A., and M. Hayakawa, Seismo Electromagnetics and Related Phenomena: History and latest results, TERRAPUB, Tokyo, 189p, 2008.

Molchanov, O. A., A. Yu. Schekotov, E. N. Fedorov, G. G. Belyaev, and E. E. Gordeev, Preseismic ULF electromagnetic effect from observation at Kamchatka, Natural Hazards Earth System Sci., 3, 1-7, 2003.

Nagao, T., Y. Enomoto, Y. Fujinawa, M. Hata, M. Hayakawa, Q. Huang, J. Izutsu, Y. Kushida, K. Maeda, K. Oike, S. Uyeda, and T. Yoshino, Electromagnetic anomalies associated with 1995 Kobe earthquake, J. Geodynamics, 33, 477-487, 2002.

Ohta, K., J. Izutsu, and M. Hayakawa, Anomalous excitation of Schumann resonances and additional anomalous resonances before the 2004 Mid- Niigata prefecture earthquake and the 2007 Noto Hantou earthquake, Phys. Chem. Earth, 34(6\&7), 441-448, 2009.

Ohta, K., J. Izutsu, A. Schekotov, and M. Hayakawa, The ULF/ELF electromagnetic radiation before the 11 March 2011 Japanese earthquake, Radio Sci., 48, 589-596, doi:10.1002/ rds.20064, 2013. 
Ouzounov, D., S. Pulinets, A. Romanov, A. Romanov, K. Tsybulya, D. Davidenko, M. Kafatos, and P. Taylor, Atmosphere-ionosphere response to the M9 Tohoku earthquake revealed by multi-instrument space-borne and ground observations: Preliminary results, Earthq. Res., 24, 557-564, 2011.

Ouzounov, D., S. Pulinets, K. Hattori, and P. Taylor (Eds), Pre-Earthquake Processes: A multidisciplinary approach to earthquake prediction studies, AGU Geophysical Monograph 234, Wiley, 365p, 2018.

Ouzounov, D., S. Pulinets, J. Y. Liu, K. Hattori, and P. Han, Multiparameter assessment of preearthquake atmospheric signals, in "Pre-Earthquake Processes: A multidisciplinary approach to earthquake prediction studies”, ed. by D. Ouzounov et al., AGU Monograph 234, Wiley, 339-359, 2018.

Parrot, M., Satellite observations of ionospheric perturbations related to seismic activity, in "Earthquake Prediction Studies: Seismo Electromagnetics”, Ed. by M. Hayakawa, TERRAPUB, Tokyo, 1-16, 2013.

Parrot, M., and M. Li, Statistical analysis of the ionospheric density recorded by the Demeter satellite during seismic activity, in "Pre-earthquake Processes: A multidisciplinary approach to earthquake prediction studies”, Ed. by D. Ouzounov et al., AGU Geophysical Monograph 234, 319-328, 2018.

Potirakis, S. M., M. Hayakawa, and A. Schekotov, Fractal analysis of the ground-recorded ULF magnetic fields prior to the 11 March 2011 Tohoku earthquake (Mw=9): discriminating possible earthquake precursors from space-sourced disturbances, Natural Hazards, doi:10.1007/s11069-016-2558-8, 2016.

Potirakis, S. M., A. Schekotov, T. Asano, and M. Hayakawa, Natural time analysis on the ultra-low frequency magnetic field variations prior to the 2016 Kumamoto (Japan) earthquakes, J. Asian Earth Sci., 154, 419-427, 2018a.

Potirakis, S. M., T. Asano, and M. Hayakawa, Criticality analysis of the lower ionosphere perturbations prior to the 2016 Kumamoto (Japan) earthquakes as based on VLF electromagnetic wave propagation data observed at multiple stations, Entropy, 20(3), 199; doi:10.3390/e20030199, 2018b.

Potirakis, S. M., P. Kasnesis, Y. Contoyiannis, N. A. Tatlas, S. A. Mitillineos, T. Asano, and M. Hayakawa, A decision making system using deep learning for earthquake prediction by means of electromagnetic precursors, Paper presented in EMSEV meeting, September, Potenza Italy, 2018c.

Potirakis, S. M., A. Schekotov, Y. Contoyiannis, G. Balasis, G. E. Koulouras, N. S Melis, A. Z. Boutsi, M. Hayakawa, K. Eftaxias, and C. Nomicos, On possible electromagnetic precursors to a significant earthquake ( $\mathrm{Mw}=6.3$ ) occurred in Lesvos (Greece) on 12 June 2017, Entropy, 21, 241; doi: 10. 3390/ e21030241, 2019.

Pulinets, S., Lithosphere-atmosphere-ionospheric coupling (LAIC) model, in "Electromagnetic Phenomena Associated with Earthquakes", Ed. by M. Hayakawa, Transworld Research Network, Trivandrum India, 235-253, 2009.

Pulinets, S., and K. Boyarchuk, Ionospheric Precursors of Earthquakes, Springer, Berlin, 315p, 2004. 
Rikitake, T., Predictions and Precursors of Major Earthquakes: The Science of Macroscopic Anomalous Phenomena, Terra Sci. Pub., Tokyo, 198p, 2001.

Rozhnoi, A., M. S. Solovieva, O. A. Molchanov and M. Hayakawa, Middle latitude LF (40 kHz) phase variations associated with earthquakes for quiet and disturbed geomagnetic conditions, Phys. Chem. Earth, 29, 589-598, 2004.

Sarlis, N. V., Statistical significance of Earth's electric and magnetic field variations preceding earthquakes in Greece and Japan Revisited, Entropy, 20, 561, doi:10.3390/e20080561, 2019.

Sarlis, N. V., E. S. Skordas, and P. A. Varotsos, Order parameter fluctuation of seismicity in natural time before and after main shocks, Europhysics Lett., 91, 59001, 2010.

Schekotov, A., and M. Hayakawa, ULF/ELF Electromagnetic Phenomena for Short-term Earthquake Prediction, LAP Lambert Acad. Pub., Beau Bassin, 102p, 2017.

Schekotov, A. Y., O. A. Molchanov, M. Hayakawa, E. N. Fedorov, V. N. Chebrov, V. I. Sinitsin, E. E. Gordeev, G. G. Belyaev, and N. V. Yagova, ULF/ELF magnetic field variations from atmosphere induced by seismicity, Radio Sci., RS6S90, doi:10.1029/2005RS003441, 2007.

Schekotov, A., E. Fedorov, O. A. Molchanov, and M. Hayakawa, Low frequency electromagnetic precursors as a prospect for earthquake prediction, in "Earthquake Prediction Studies: Seismo Electromagnetics”, Ed. by M. Hayakawa, TERRAPUB, Tokyo, 81-99, 2013.

Schekotov, A., and M. Hayakawa, Seismo-meteo-electromagnetic phenomena observed during a 5-year internal around the 2011 Tohoku earthquake, Phys. Chem. Earth., Parts A/B/C, doi:10.106/j.pce.2015.01.010, 2015.

Smirnova, N., M. Hayakawa, and K. Gotoh, Precursory behavior of fractal characteristics of the ULF electromagnetic fields in seismic active zones before strong earthquakes, Phys. Chem. Earth, 29, 445-451, 2004.

Sorokin, V. M., and M. Hayakawa, On the generation of narrow-banded ULF/ELF pulsations in the lower ionospheric conducting layer, J. Geophys. Res., 113, A06306, doi:10.1029/2008JA013094, 2008.

Sorokin, V., and M. Hayakawa, Plasma and electromagnetic effects caused by the seismic-related disturbances of electric current in the global circuit, Modern Applied Science, 8(4), 61-83, 2014.

Sorokin, V., V. Chmyrev, and M. Hayakawa, Electrodynamical Coupling of Lithosphere-AtmosphereIonosphere of the Earth, NOVA Publishers, New York, 351p, 2015.

Tramutoli, V., N. Genzaro, M. Lisi, and N. Pergola, Significant cases of preseismic thermal infrared anomalies, in "Pre-earthquake Processes: A multidisciplinary approach to earthquake prediction studies", Ed. by D. Ouzounov et al., AGU Monograph 234, Wiley, 331-338, 2018. 
Tronin, A. A., M. Hayakawa, and O. A. Molchanov, Thermal IR satellite data application for earthquake research in Japan and China, J. Geodynamics, 33, 477-487, 2002.

Uyeda, S., and T. Nagao, International cooperation in pre-earthquake studies: History and new directions, in "Pre-earthquake Processes: A multidisciplinary approach to earthquake prediction studies", Ed. by D. Ouzounov et al., AGU Geophysical Monograph 234, Wiley, 3-6, 2018.

Uyeda, S., T. Nagao, K. Hattori, Y. Noda, M. Hayakawa, K. Miyaki, O. A. Molchanov, V. Gladychev, L. Baransky, A. Schekotov, G. Belyaev, E. Fedorov, O. Pokholetov, S. Andreevsky, A. Rozhnoi, Y. Khabazin, A. Gorbatikov, E. Gordeev, V. Chebrov, A. Lutikov, S. Yunga, G. Kosarev, and V. Surkov, Some preliminary results of seismo-electromagnetic research at complex geophysical observatory, Kamchatka, in "Seismo Electromagnetics: Lithosphere-Atmosphere-Ionosphere Coupling," Ed. by M. Hayakawa, and O. A. Molchanov, TERRAPUB, Tokyo, 413-419, 2002.

Varotsos, P., The Physics of Seismic Electric Signals, TERRAPUB, Tokyo, 338p, 2005.

Varotsos, P., and K. Alexopoulos, Physical properties of the variations of the electric field of the earth preceding earthquakes, I \& II, Tectonophys., 110, 73-125, 1984.

$\mathrm{Xu}$, G., P. Han, Q. Huang, K. Hattori, F. Fabriani, and H. Yamaguchi, Anomalous behavior of geomagnetic variations prior to the 2011 off the Pacific coast of Tohoku earthquake (Mw9.0), J. Asian Earth Sci., 77, 59-65, 2013.

Yang, S. S., T. Asano, and M. Hayakawa, Abnormal gravity wave activity in the stratosphere prior to the 2016 Kumamoto earthquakes, J. Geophys. Res., Space Physics, 124, http://doi.org/10.1029/2018JA026002, 2019.

Yasuda, Y., Y. Ida, T. Gotoh, and M. Hayakawa, Interferometric direction finding of over-horizon VHF transmitter signals and natural VHF radio emissions possibly associated with earthquakes, Radio Sci., 44, RS2009, doi:10.1029/2008RS003884, 2009. 